\title{
Salivary Hormones, Immunes and Other Secretory Substances as Possible Stress Biomarker
}

\author{
Shusaku Nomura \\ Nagaoka University of Technology, \\ Japan
}

\section{Introduction}

In the modern society, the issues on mental health have always been pressing and global problem, and unfortunately it remains today unsolved. It does not apply only to a personal matter, but also having a huge impact on economics as it has estimated a decade ago of that the social cost concerning mental health problem in European Union was 3 to $4 \%$ of GNP (Gabriel, 2000). However generally speaking, the mental stress is quite difficult to be aware of personally. It is hard for anyone to manage mental stress by on his/her own. Therefore it is an urgent task to figure out a "practical" methodology to evaluate, manage and control the mental stresses.

On the other hand, recent developments of the molecular analysis techniques has been revealed that particular hormones and immune substances secreted within human body change its level in responding to human mental state. For an instance, salivary Immunoglobulin-A shows a transient increase against short-term psychological stressors such as mental arithmetic task, stroop task, academic presentation (Valdimarsdottir, 1994). "All illnesses come from the mind" is no longer a folk story. These particular hormones and immune substances can potentially be a practical biomarker for human mental stress. Number of hormones and other substances in our body has been studied as a possible stress biomarker (Izawa, 2004; Wakida, 2004), and the number of academic research has been increasing as well, as shown in Figure 1. Currently it forms an interdisciplinary research field called psychoneuroendocrinology (PNE) and/or psychoneuroimmunology (PNI) (Ader, 2001) (hereafter, we use the term psychoneuroendocrine-immunology (PNEI) to indicate both PNE and PNI).

PNEI must be a contributory research field which should possibly establish "practical" criteria for objectively evaluating human mental state. However it is a relatively new field of study still developing, there are a lot of stuffs to be investigated, e.g. the precise stress response of these biomarkers in the time series, the sensitivity of the response against other than acute stressors, physiological mechanism dynamically regulating the release of these biomarkers in responding to stress.

In this chapter, PNEI research of its background, method, experiments, and mathematical modelling approach are introduced. 


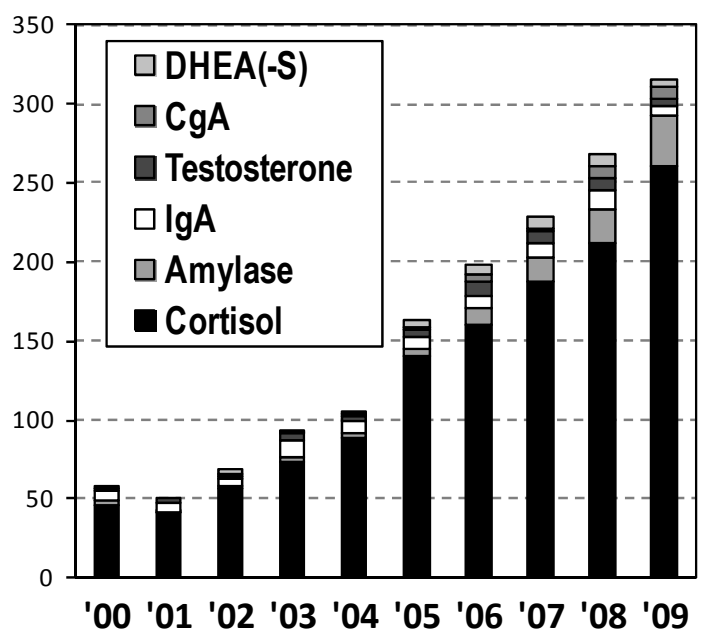

Fig. 1. Number of salivary biomarker researches: Retrieved by "PubMed" with queries "saliva", "stress" and "X (one of each substance)"

\section{Stress response in our body: SAM and HPA system, and possible biomarkers}

In the long history of stress research on human or animals, it has been revealed the existence of two internal stress reaction physiological pathways namely: (1) hypothalamus-pituitaryadrenal (HPA) and (2) sympatho-adrenal-medullary (SAM) system (Kirschbaum, 1994) as shown in Figure 2. When one perceives a stressful situation subjectively, one of or both SAM and HPA system is activated resulting a sort of cascade chain-reaction of a variety of biochemical substances inside our body along with SAM and HPA axes. During such a cascade chain-reaction, wide variety of bio-chemical substances, such as hormones, proteins, and peptides, were released into blood stream or other secretory fluid, such as saliva, urine, breast milk, sweat, etc. Therefore by monitoring such stress-related substances, the activation of SAM and/or HPA system, or nature of perceived stressor is expected to estimate to some extent. This is the background idea.

In the current PNEI studies, mainly seven biomarkers falling under the categories of hormones, immune substances, proteins and enzymes; Immunoglobulin A (IgA), cortisol, human Chromogranin A (CgA), alpha-amylase, Dehydroepiandrosterone (DHEA), Dehydroepiandrosterone sulfate (DHEA-S), and testosterone (TE), has been frequently employed (Deguchi, 2006; Bosch, 2002; Michael, 2000; Nakane, 1999; Kirschbaum, 1994). IgA, CgA, and amylase are considered to reflect SAM activation, and cortisol, DHEA, DHEA-S, and TE are considered to reflect HPA activation. The goal of PNEI study is to investigate the activations of these two systems under various stressful situations using secretory hormones and immune substances, and to demonstrate the congruity of these substances as an objective measurement of human mental stress. In the following contents, these biomarkers are described with reviewing past PNEI studies. 

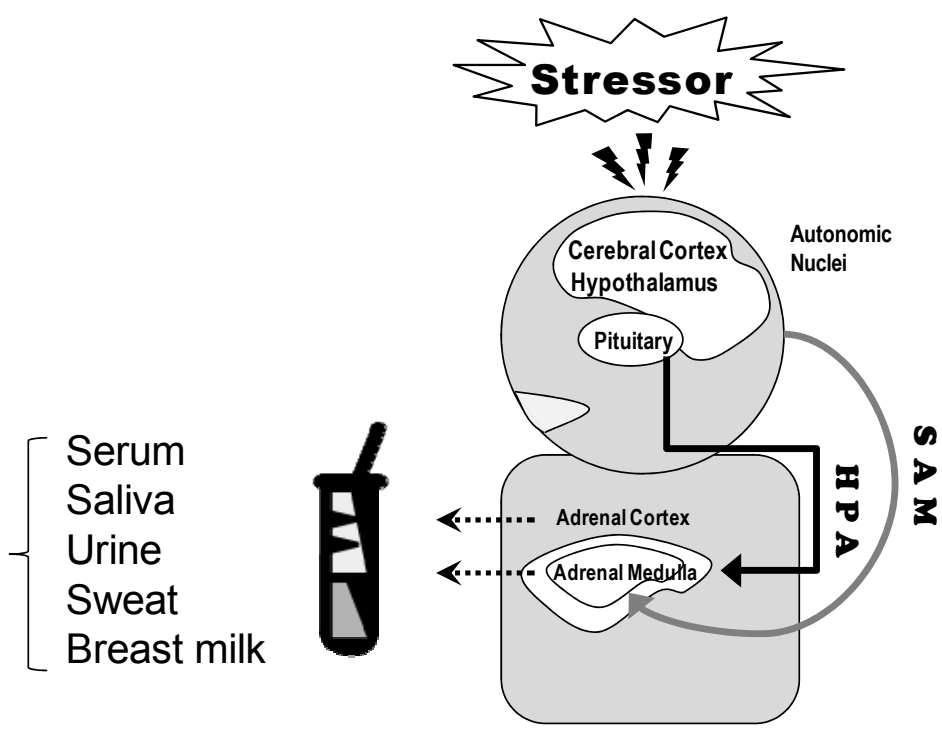

Fig. 2. Stress reaction physiological pathways: HPA and SAM system

\subsection{Salivary Immunoglobulin A $(\lg A)$}

An antibody, Immunoglobulin A (IgA), is one of the most important substances in human immune system (Tsujita, 1999). It is present in almost all human secretory fluids, such as saliva, serum, urine, breast milk etc. IgA works non-specifically and therefore plays a very important role on our health, e.g. preventing bacteria from forming colonies, neutralizing toxins and enzymes produced by bacteria, and inhibiting pathogenic viruses from penetrating into the epithelial cell. Especially the salivary IgA called as "the first line of defense" since it functions in the mouse to prevent from contracting influenza or other respiratory tract infection (URTI) illnesses. In fact, clinical studies have suggested the negative correlation between the level of salivary IgA and the incidence of an acute URTI (Jemmott III, 1989). It was also reported the relevance between the level of salivary IgA and caries or periodontitis (Gregory, 1992).

On the other hand, it has been reported that salivary IgA changes its level in response to various psychological factors (Bosh, 2002), such as desirable or undesirable daily events (Stone, 1994), daily hassles (Martin, 1988), negative or positive moods (Martin, 1993), academic stresses such as examination (Jemmott III, 1983) and presentation (Evans, 1994), a short-term stressful cognitive task (Jemmott III, 1989), and relaxation (Green, 1987; Knight, 2001). These stress responses of IgA can be classified into two types: 1) a transient increase of IgA secretion immediately after an exposure of short-term laboratory stressor and subsequent recovering to the basal lever after the removal of the stressor, and 2) decline of basal IgA level several days after or during a long-term stressful situation or under chronic stress. The transient increase of IgA is considered to be regulated by autonomous nervous system (Valdimarsdottir, 1997). Thus it can be taken as a biomarker for SAM system activitiy. The decline of basal IgA level over longer period of time, by contrast, is considered to indicate chronic wear and tear of production capability. Thus it is assumed to represents 
some sort of long-term or chronic mental stress state while it is not necessary to consider in relation to HPA system modulation.

Although the transient increase of IgA by laboratory stressors has been consistently observed in the past PNEI studies, the decline of basal IgA over longer period of time according to chronic stress has not been always observed. A review paper on IgA studies pointed out that studies targeting on chronic stress had methodological defects such as less control of subjects' physical conditions e.g. sleep and diet, variety of saliva sampling methods, and introducing non-standardized psychological questionnaires (Bosh, 2002). Moreover the transient increase of IgA would easily mask the change in the basal IgA level over long period of time: if a subject got nervous at the moment of saliva sampling, the observed IgA level would no longer a "basal" level of the IgA of the sampling day.

There are also IgA studies focusing on the effects of various relaxing factors. These studies also reported a transient increase of IgA as the same as studies focusing on laboratory stressors. However few attempts have been made to investigate the effects of such relaxing factors with/under stressful situation (Valdimarsdottir, 1997), so it is not clear whether such a transient increase of IgA induced by relaxing factor would be derived from the same physiological mechanism as the response against laboratory stressors.

Other IgA studies have revealed that the higher cognitive process could mediate the IgA secretion. Psycho-social support alleviated the IgA secretion under long-term (Jemmott III, 1988) and short-term (Ohira, 2004) stressors. Subjects categorized in Type A trait, who are typically represented as short tempered and strong hostile, showed higher baseline of IgA and lower reactivity of IgA against an acute stressor (Ohira, 1999). Controllability of a given stressor unconsciously determined the salivary IgA (Ohira, 2001). These studies suggest that the higher cognitive process could mediate the IgA secretion. However the number of studies targeting on these potential mediators remains small.

Altogether IgA can be a useful stress biomarker, especially for laboratory stressors, representing SAM system activation, while the change in the basal IgA level over long period of time is still be a matter in discussion.

\subsection{Salivary cortisol}

Cortisol is the most potent glucocorticoid produced and secreted from adrenal cortex playing a quite important role for maintain our body, e.g., keeping blood glucose level adequately. Cortisol levels can be measured in serum, urine, and saliva. Cortisol is considered to be released into blood stream via activation of HPA system. In addition significant positive correlation has been obtained between salivary and blood cortisol. Therefore salivary cortisol is assumed as a possible stress biomarker representing HPA system activity.

In the past cortisol studies, a transient increase of salivary cortisol was observed by shortterm laboratory stressors, such as mental arithmetic task, stroop task, and oral presentation (Dickerson, 2004; Kirschbaum, 1994). With regard to a long-term or chronic stress, higher level of cortisol secretion has been revealed to associate with chronic stress state, such as job stress (Steptoe, 2000), job loss (Ockenfels, 1995), and divorce (Powell, 2002). By contrast positive affect was found to associate with lower total cortisol secretion during daytime 
(Steptoe, 2005). Cortisol has a large diurnal variation of which it starts increase at the just after awakening in the morning, reaches at the peak of the day around 30 minutes after awakening, keeps decreasing through the daytime, and then reaches a minimum level at the night. Especially the individual drastic change, increasing to the peak of the day at the morning is called "cortisol awakening response (CAR)" and it has reported that CAR is associated with chronic stress (Izawa, 2007; Clow, 2004; Schulz 1998; Pruessner, 1997). Altogether salivary cortisol can be a possible short-term and long-term stress marker.

However cortisol has been consistently reported to increase against such stressors accompanying with strong tension and threat, or psycho-social evaluation, it showed inconsistent results against relatively mild stressors such as simple arithmetic task and cognitive task (Dickerson, 2004). Some methodological defeats have been discussed on this discrepancy such as variety of biochemical analysis, saliva collection method, and subject control. Among that, the variety of the timing of saliva sampling has been frequently indicated, including the past PNEI studies with other biomarkers (Hansen, 2008; Dickerson, 2004; Bosch, 2002; Valdimarsdottir, 1997; Kirschbaum, 1994). Also it should be noted that because the stress reaction of HPA system is truly complicated and potentially mediated by variety of physiological factors, the salivary cortisol cannot be taken as a direct measure of HPA system itself but as rather an "indirect" measure (Hellhammer, 2009).

\subsection{Salivary $\mathrm{CgA}$}

Chromogranin A (CgA), a major member of the granin family of acidic secretory glycoproteins, is known to be released from the adrenal medulla into the blood with catecholamine(Kim, 2005). Therefore it is considered to be a possible biomarker of SAM system (Nakane, 1999; Winkler, 1992). CgA has been reported to transiently increase in response to short-term laboratory stressors such as a calculation test (Nakane, 1999), white noise (Miyakawa, 2006), and a cognitive task (Kanamaru, 2006). On the other hand there are studies failed to observe increase or rather decrease against a laboratory stressors (e.g., Yamakoshi, 2009) and observed a transient increase in subjects who declared the positive affection after watching a comic video (Toda, 2007).

$\mathrm{CgA}$ is expected as a possible stress marker representing SAM system activity as just described. However, the number of studies on salivary CgA is still small comparing with that on Cortisol and IgA. Moreover commercial available assay protocol for CgA is limited as just one product (Human Chromogranin A EIA Kit, Yanaihara Institute Inc.). So is necessary to accumulate more studies for further discussion.

\subsection{Salivary DHEA}

Dehydroepiandrosterone (DHEA) and Dehydroepiandrosterone sulfate (DHEA-S) is a steroid adrenal cortex hormone like cortisol. It is thus expected as a possible stress marker representing HPA system activity. It is considered to function antagonistically with cortisol on the central nervous system and immune system (Wolf, 1999). DHEA and DHEA-S was reported to associate with superior stress tolerance (Morgan, 2009). On the contrary lowered DHEA was observed in subjects with partially or completely remitted depression (Michael, 2000). Therefore higher DHEA can be considered in relation with positive state in the context of chronic stress. 
It was also reported to show a transient increase against short-term laboratory stressors like as other possible biomarkers (Shirtcliff, 2007; Sugaya, 2007; Izawa, 2008). However these studies introduced a strong and socially evaluated laboratory stressor, as typified by "Trier Social Stress Test (TSST) (Kirschbaum, 1993)," responses against relatively mild stressors such as simple arithmetic task and cognitive task is unknown.

Although DHEA in many respects paralleled cortisol secretory activity there was some dissociation in the response in the time series (Sugaya, 2007) and diurnal secretion (Hucklebridge, 2005). DHEA studies are also still small in number as depicted in Fig.1. Further studies are necessary to discuss more.

\subsection{Other salivary possible biomarkers}

Salivary alpha-amylase (sAA) has been reported to show a transient increase against shortterm laboratory stressors along with the activation of sympathetic nervous system mediated via beta-receptor (van Stegeren, 2006; Deguchi, 2006; Yamaguchi, 2004;). Therefore it is also considered to be a possible biomarker representing SAM system activity (Bosch, 2002). However it is suggested that the activation of parasympathetic nervous system also results in a transient increase of sAA mediated via increment of saliva flow rate (Bosch, 2002).

Regarding with chronic stress sAA was reported to have no remarkable association with depression, anxiety, work stress and burnout but a small negative correlation between "social difficulties" measured with a chronic stress scale in a population of nurses (Wingenfeld, 2010). Recently alpha-amylase is used to highlight the difference in the activity of SAM system and HPA system which is measured by salivary cortisol (Strahler, 2010), and a study reported that the salivary alpha-amylase over cortisol ratio, named as $\mathrm{AOCg}$, can be a better indication of stress system dysregulation than sAA or cortisol alone (Ali, 2011).

Free-3-mehoxy-4-hydroxyphenylglycol (free-MHPG) (Okamura, 2010; Buchsbaum, 1981) and testosterone was reported to show transient increase by short-term laboratory stressors, while the number of studies assessing these substances are very small and frequently showed inconsistent results (e.g. Schoofs, 2011).

\subsection{Summary of possible biomarkers}

In the current PNEI studies, it is suggested that the salivary biomarkers can be taken as a possible objective stress measurement. All abovementioned substances show a transient increase against short-term laboratory stressor representing the activation of human physiological stress reaction pathways, HPA and SAM systems. Cortisol is the most promising biomarker for a long-term or chronic stress, and IgA is the one for short-term laboratory stressors. However, since PNEI is relatively a new interdisciplinary study, basic problems remain unsolved such as sensitivity of these biomarkers against variety of stressful and relaxing factors, precise change in the secretion of biomarkers in the time series, the effects of long-term stress or chronic stress which might affect the change in the production speed of these biomarkers rather than a temporal change in the secretion.

A recent PNEI research for assessing all different types of biomarkers in blood, such as active natural killer (NK) cell level, varieties of T lymphocyte, dopamine, norepinephrine, and epinephrine, shows a rapid change in the composition of those substances (e.g., Kimura, 
2005; Isowa, 2004). It provides quite important information for the better understanding of the human psycho-physiological stress reaction. However such a multi-assessing study is still at its infancy. Far more research needs to be conducted for more discussion.

\section{Methods of PNEI Studies using saliva samples}

In this chapter, the methodology of PNEI studies, i.e. experimental designs, subjects' control, preparation of stressors, analysis of biomarkers etc., is described since the variety of methodology has frequently been pointed out as one of a major confounder.

\subsection{Saliva sampling method}

Saliva samples have been collected frequently by "Salivette", which is made of dense plain cotton of a cylindrical shape about $1 \mathrm{~cm}$ wide and $3.5 \mathrm{~cm}$ long. Salivette is designed for onetime saliva sampling and mostly introduced in diagnosis uses. In other words, it is not suitable for repetitive saliva collection. It has high absorbability and thus deprives far more amount of saliva, about $2 \mathrm{~mL}$ per one sampling for 3 minute, for that of necessary to quantitative determination of biomarkers, at most $50 \mu \mathrm{L}$ of saliva for one biomarker. Excessive saliva collection brings forth the lack of saliva and, as a result, disturbs normal saliva flow. A past study demonstrated that repetitive saliva collection in every 5 minutes resulted in the decrease of saliva volume and also the concentration of salivary IgA by sampling time as depicted in Fig.3 (Nomura, 2006). Therefore the use of Salivette in case of repetitive sampling is not recommended.

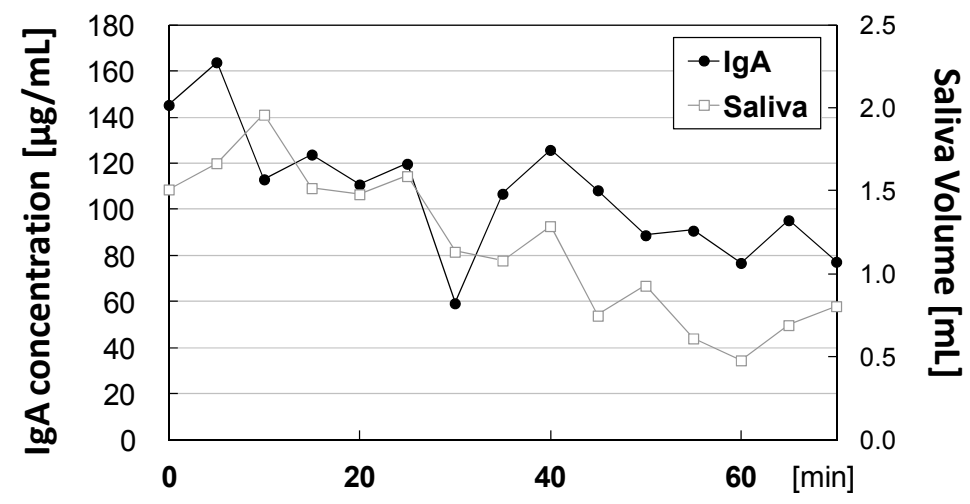

Fig. 3. The change in IgA concentration and saliva volume with repetitive saliva collection by Salivette

The passive drawing or the use of small cotton is recommended in case of repetitive sampling. Considering the absorption of biochemical substances by the cotton, the passive drawing is technically ideal method for saliva sampling. However it requires training for subjects to get used to dropping saliva into small cup or container. Besides it might be uncomfortable for some subjects to take saliva in this way. Taking saliva by small cotton is easiest method. Although a certain amount of biomarkers would be absorbed, it might be excluded when one focuses on the relative change in the level of biomarkers. It is important 
to prepare the same size and volume of cotton for the repetitive sampling otherwise the volume of saliva collection would become as an unintended confounder. The small cotton should be placed under the subjects' tongue so as to collect the fresh saliva.

Experimenter should pay attention in handling the sample saliva to avoid contingent infection of virus or other possible diseases. All experimenters should ware disposable gloves and glasses. The saliva samples should be kept in the freezer below -20 Celsius by the day of the quantitative determination of biomarkers.

\subsection{Possible confounders}

The natural secretion of abovementioned biomarkers has a diurnal change: the highest level is in the morning and gradually decreases afterwards to the lowest level in the night time (it should be noted that only alpha-amylase act controversy; Strahler, 2010). Therefore saliva sampling should be conducted depending on the objective of a study: (1) it should be corrected in the afternoon where the secretion of biomarkers is expected to be stable in the case of laboratory stressor studies by which a level of transient increase of these biomarkers are focused, and (2) in the morning or just after awakening in the case of chronic stress study since basal secretion level is expected to show a remarkable difference among subjects in the morning (e.g. Steptoe, 2005). Because of its remarkable diurnal change, repetitive sampling by a distinct time point of a day is recommended even in a chronic stress study.

Postprandial effects on the secretion of biomarkers are considerable. In addition $\mathrm{pH}$ of saliva could affect the quantitative determination of biomarkers. Subjects should not take any food or drink except for water at least an hour prior to the experiment. Hard exercise should be avoided prior to the experiment since it could make biomarkers temporary elevate. Moreover it is strongly recommended to take 5 to 10 minutes of an initial rest period before conducting experiment. If subjects were not familiar with or nervous to an experiment, most of biomarkers would increase by such a negative feeling or strain. Subjects who take any medications, especially oral contraceptive, suffer from any disease, are pregnant, or other cases in which physiological states were considered to be unusual should be excluded. Age and gender should be balanced in groups of between-subjects study since it is known to be confounders, especially for the study assessing hormones.

Subjects must be well informed about the objective and method of the study. Any experimental design targeting on human mental or physical stress should be approved by a local ethics committee or equivalent organization.

\subsection{Quantitative determination of salivary biomarkers}

The concentrations of salivary biomarkers have frequently been determined by enzymelinked immunosorbent assay (ELISA). ELISA is nowadays one of major molecular determination techniques. It is much easier in treatment and cheaper in running cost than other molecular determination techniques, such as radioimmunoassay (RIA), fluorescent immunoassay (FIA), and the high performance liquid chromatography (HPLC). Several products which are designed for determination of "salivary" biomarkers, not for serum and urine, are placing on the market (e.g., Salivary Secretory IgA Indirect Enzyme Immunoassay Kit, Salimetrics, LLC., USA). By using such an ELISA kit in which all specimens and 
materials are included, experimenter can easily assay various biomarkers with a minimum knowledge of biochemical analysis.

The principle of ELISA is based on the antigen-antibody reaction which is for capturing a target substance and the enzyme reaction which is for detecting the mass of a target substance via optical density of reaction produced color. The brief description of ELISA (competitive method) is as follows: (1) Thaw saliva samples kept in a biological freezer by moving them into a biological refrigerator (4 Celsius). (2) Centrifuge each saliva samples for 10 minutes at $1500 \mathrm{rpm}$ to precipitate mucins or other solid contents. (3) Add each saliva sample (or known samples for references) into antibody-coated 96-well micro-plate. (4) Add a constant amount of "enzyme conjugate" which is the target biomarker (antigen) combined with horseradish peroxidase (HRP) into the micro-plate and incubate for an hour. In this step, antigen-antibody reaction is occurred competitively between original target in the saliva sample and that in the enzyme conjugate. (5) Wash the micro-plate to flush unbind target. (6) Add tetramethylbenzidine (TMB) solution to induce enzyme reaction with enzyme conjugate which is captured by antigen coated on the bottom of each well of the micro-plate in the step (4). The amount of the bind enzyme conjugate, which is there as a result of competitive reaction process, can be detected as the strength of optimal color $(450 \mathrm{~nm})$ cased by enzyme reaction. Therefore this optimal density is inversely proportional to the concentration of target containing in the original saliva sample. (7) Finally, the target concentration in each sample is determined by referencing the optimal density of the reference samples. All analysis procedures take roughly about 3 to 5 hours for one microplate. Correct handling of samples and specimens with well calibrated micropipette is critical for all steps.

\section{Sensitivity in the response of biomarkers against laboratory stressors}

In this section, the transient response of HPA and SAM above-mentioned stress biomarkers against a shot-term laboratory stressor is described with reviewing our past research (Nomura, 2006, 2009, 2010a, 2010b) and also additionally presenting some new experimental results. Especially, the transient response in the time series, i.e. the changing in the level of biomarkers in a short period of time through the onset or the end of an acute laboratory stressor, is featured. It can be highlighting the time constant of the physiological stress response of these biomarkers, or more simply the "temporal sensitivity" against the stressor.

As already described variety of salivary secretory substances are considered to be possible biomarkers as representing the activation of HPA and SAM system. Moreover there seems to be a difference in the "temporal sensitivity" among there biomarkers. Some studies demonstrated the difference in the time course response of these biomarkers against laboratory stressors. Izawa et al. (2008) demonstrated that the stress-induced transient DHEA increase took place ahead of that of cortisol in the TSST experimental session. Ali et al. (2011) also demonstrated in the same TSST session that alpha-amylase also precede cortisol response.

On the other hand whether there would be a difference in the "sensitivity of the intensity" among these biomarkers is still in the matter of discussion. A review article on cortisol described that the salivary cortisol increases against stronger and socially evaluated laboratory stressor, as typified by TSST, but have shown inconsistent results against 
relatively mild stressors such as simple arithmetic task and cognitive task by which IgA should always increase (Dickerson, 2004). Actually the idea of "sensitivity of the intensity" cannot stand separately from the "temporal sensitivity." The response of these biomarkers against laboratory stressors always takes place in a transient manner in a certain period of time: it gradually increases, reaches a peak, and after the removal of the stressor, it gradually decreases to the basal level. In fact, if such a transient stress-induced increase of a biomarker took place with a different time delay with respect to each biomarker, it would appear as the difference in the sensitivity of the intensity depending on the time point. Moreover in the case of that saliva sample were not taken frequently enough in the time series, it would result in the inconsistency of obtained experimental results and interpretations among the studies.

With an eye on this point, we designed an experiment to clarify the difference in the sensitivity of biomarkers against a relatively mild laboratory stressor with frequent saliva collection in the time series, as described in the next.

\subsection{Experiment targeting on the difference in the sensitivity among HPA and SAM biomarkers against a mild stressor}

The precise changing in the level of salivary four stress biomarkers, which were IgA, cortisol, CgA, and DHEA, were assessed continuously in the time series during which a simple calculation task was given to subjects as a mild stressor. The difference in the sensitivity of these biomarkers as a stress biomarker was expected to be illustrated. Moreover the better understanding for the dynamics of physiological stress response was expected as well since these four biomarkers represent HPA (cortisol and DHEA) and SAM ( $\operatorname{IgA}$ and $\mathrm{CgA}$ ) system activities respectively.

\subsubsection{A stressor}

Subjects, ten university male students, were instructed to conduct a simple calculation task as a mild stressor. It was a simple addition of two double-digit integers repeatedly presented on a laptop monitor every 3.0 seconds with changing the figures. It is quite similar to so-called Kraepelin psychodiagnostic test, which is typified as simple, monotonous, and boring arithmetic task, and thus frequently introduced for studies on mental work load or mental fatigue. Subjects were instructed to perform this calculation task as correct and fast as possible.

\subsubsection{Procedure}

Two types of experimental design of task/break schedule were prepared for this study as depicted in Figure 4. The experimental session A consists of two sets of 18 minutes of the calculation task and subsequent 9 minutes of the break. The experimental session B consists of six sets of 6 minutes of the task and subsequent 3 minutes of the break. It should be noted that the total duration of the task and break is exactly the same in both sessions: 36 minutes of the calculation task and 18 minutes of the break. After each session A and B, 20 minutes of recovering period was added during which subjects were sitting calmly on the chair.

Saliva samples were taken by a piece of cotton every three minutes during the session. Saliva was also taken at 3, 10, and 20 minutes after the last set of the task/break to observe a 


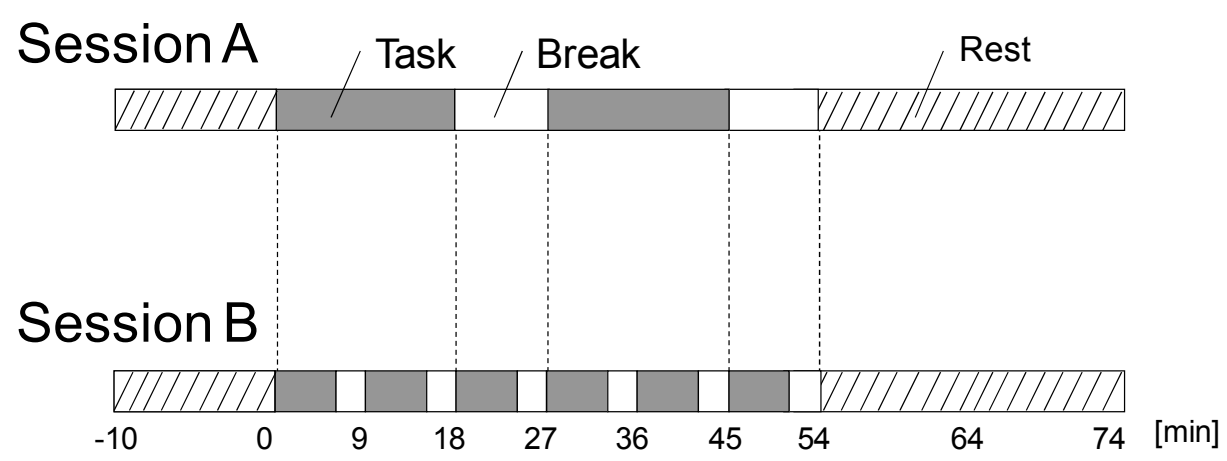

Fig. 4. Task/break schedule in session A and B

recovering process of the elevated biomarkers to a basal level. Subjects were instructed to place the cotton under his tongue for three minutes. These cottons were centrifuged at 1500 rpm for 10 minutes to remove mucin. This filtrated saliva samples were stored separately in a small polypropylene tube in freezing chamber at -20 Celsius by the day on the quantitative analysis. Four above-mentioned biomarkers, which are IgA, cortisol, CgA, and DHEA, were assessed by ELISA.

This experiment was conducted as with-in subjects design: every subject went through both sessions $\mathrm{A}$ and $\mathrm{B}$ in a randomized order. All sessions were conducted in a dark and soundproof room, one by one, and in the afternoon so as to avoid a disturbance by the large diurnal change of biomarkers in the morning.

\subsubsection{Other physiological and psychological measures}

Electrocardiogram (ECG) was recorded through all sessions and subsequent 20 minutes of recovering period by a multipurpose bio-signal amplifier at the rate of $500 \mathrm{~Hz}$ (BMS-3201, Nihon-Kohden Co.). Frequency analysis was conducted afterward to estimate the high frequency power of the ECG data in the range of 0.15 to $0.40 \mathrm{~Hz}$ (hereafter denoted as HF power), which has been frequently used for the index of parasympathetic nervous system activity.

Regarding with the psychological scale, "Profile of Mood State (POMS) (Japanese version)" (Yokoyama, 1993) were given to the subjects to fill up before and after the sessions. POMS is one of the most commonly used questionnaires frequently used various psychological and cognitive science studies (MacNair, 2003). It consists of 65 items concerning subjects' mood state with 5 point scale: not at all, a little, moderately, quite a lot, and extremely. These items are designed to classify into six identified mood factors: tension-anxiety (defined as T-A), depression-dejection (D), anger-hostility $(\mathrm{A}-\mathrm{H})$, fatigue-inertia $(\mathrm{F})$, vigour-activity $(\mathrm{V})$, and confusion-bewilderment $(\mathrm{C})$. The score of each mood factors can be found by adding up the scores of corresponding items. 


\subsection{Results of the experiment: The difference in the sensitivity among HPA and SAM biomarkers}

\subsubsection{Behavior and POMS}

The task performance did not show any change by repetition of the task during sessions or between the session A and B. In fact the task, which is the addition of two double-digit integers at every 3.0 seconds, is too easy for the subjects, who were university students, to find any difference in their performance.

Regarding with POMS, only the factor V (vigour-activity) out of six mood factors showed significant decrease after the calculation task $(p<.05, t$-test). Other factors did not show any significant change in the scores while the factor $F$ (fatigue) showed a trend of decrease $(p<.1)$. In other words, the calculation task introduced in our study was not an intensive stressor which gives subjects negative mood such as tension, threat, or hostility just like TSST but rather a mild stressor as we intended.

\subsubsection{Profile of autonomous nervous system activities (HR, HF power of ECG)}

Figure 5 shows the profile of changing in the heat rate (HR) during task/break period in the time series in the session A (Fig. 5(a)) and B (Fig. 5(b)). It should be noted each values were found by averaging every three minutes so that it makes easier to illustrate the difference in the profile with that of biomarkers as depicted later. There was no any significant change in the time series regardless of task, break, or recovering period in both session A and B, while it has a tendency to decrease in the task period. It is known through the past experimental psychological researches that the heart rate frequently decreased rather than increased under a certain type of laboratory stressors by which subjects need to concentrate on or just keep silence in perceiving the situation, such as vigilance task, noise exposure, mental arithmetic task, etc (Williams, 1986). At any rate this results again ensure the nature of the calculation task as a mild stressor as we intended.
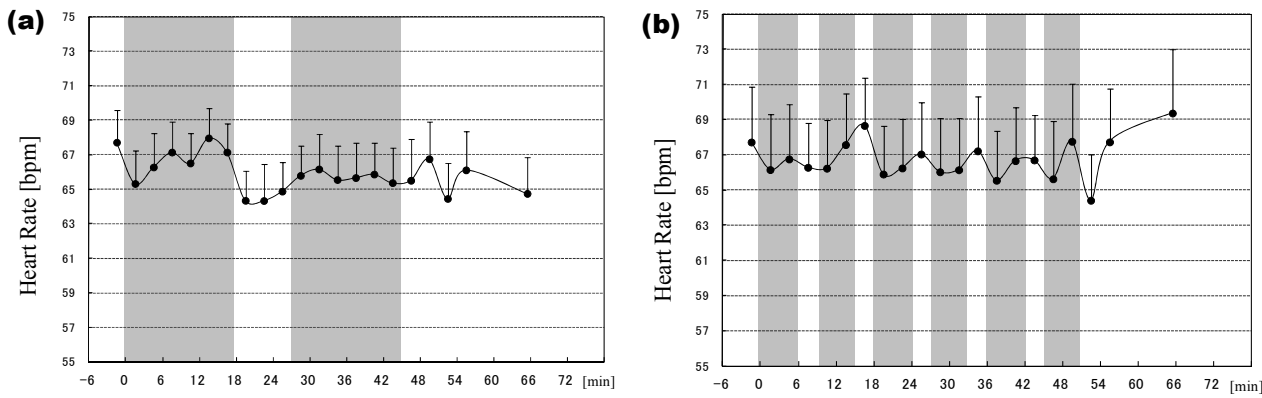

Fig. 5. Profile of heart rate in session A and B. Error bar represent standard error of the mean (S.E.M). Gray background represents a calculation task period.

Figure 6 shows the profile of changing in the high frequency power of ECG signal (HF power) during task/break period in the time series in the session A (Fig. 6(a)) and B (Fig. 6(b)). As these figures shows, HF power remarkably decreased during the task and recovered to the basal level in the subsequent break period. HF power changes according to 
respiratory regulated heart beat-to-beat interval modulation, which is so-called Respiratory Sinus Arrhythmia (RSA) (Andreassi, 2007). RSA is dominantly subject to the parasympathetic nervous system, so HF power is considered as an index of the parasympathetic nervous system activities. When one look at the results of our study, it is quite understandable since HF power decreased during the task and recovered in the rest period. Moreover it should be emphasized that such switch-over of the HF power took place rapidly according to the task/break schedule, and well reproduced over time regardless of the repetition of the task/break as seen the session B in particular. Therefore with regard to the "sensitivity" of HF power against a mild stressor, HF power can be compared with a binary switch as it turn on during the task and off in the break without any cumulative effect over time.
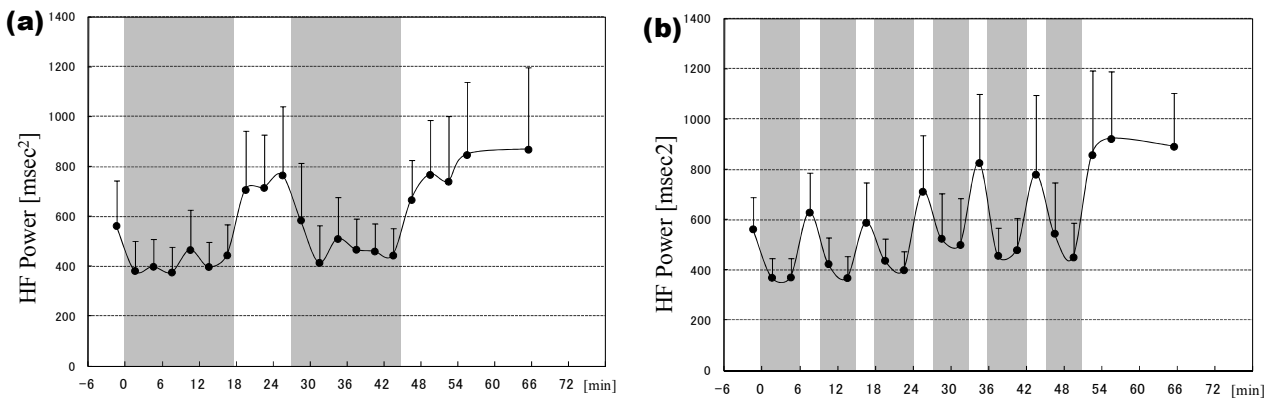

Fig. 6. Profile of HF power of ECG in session A and B. Error bar represent standard error of the mean (S.E.M). Gray background represents a calculation task period.

\subsubsection{Profile of $\operatorname{Ig} \mathrm{A}$}

Figure 7 shows the profile of changing in the concentration of salivary secreted IgA during task/break period in the time series in the session A (Fig. 7(a)) and B (Fig. 7(b)). It increased during the task, decreased during the break, and recovered to the initial (basal) level. This simple fact demonstrates the congruity of IgA as a stress biomarker of a mild stressor.
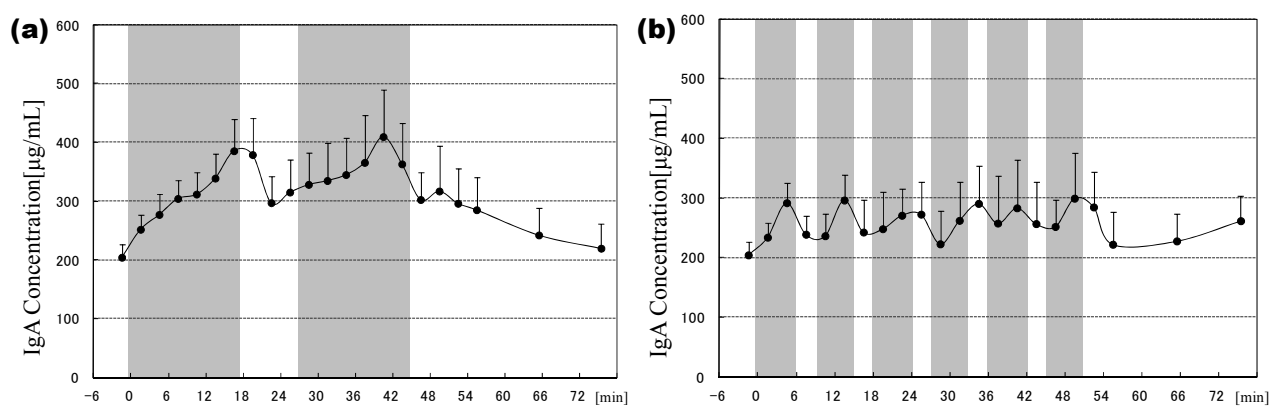

Fig. 7. Profile of IgA in session A and B. Error bar represent standard error of the mean (S.E.M). Gray background represents a calculation task period. 
On the other hand IgA showed a remarkable difference in the profile over time comparing with that of HF power. In session A, IgA started to increase gradually after the onset of the first calculation task. Following the end of the first task, IgA started to decreased but because of certain latency in recovering, it did not recover to the basal level. These latencies over time in IgA increasing induced by the onset of a stressor and decreasing in the recovering resulted in cumulative profile of $\operatorname{IgA}$ as depicted in Fig. 7(a): where the average concentration of IgA during the first task period was significantly higher than that of the second task $(p<.01, t$-test), and it took around 30 minutes for the elevated IgA to return to the basal level. Moreover there was no significant correlation between IgA concentration and saliva flow rate. Therefore the cumulative effect observed in session A was not merely as a result of the change in saliva flow, which is thought to be mediated by the change in autonomous nervous system activity.

Comparing with the results in session $\mathrm{A}$ and $\mathrm{B}$, there was no such remarkable cumulative effect in session B: there was no any difference in the initial (basal) level, but regarding with the total IgA secreted during all through the sessions, it was significantly higher in session A than that of B $(p<.01)$. This difference in the IgA secretion profile in the session A and B is rather intriguing, because the total task/break period, and moreover the task/break ratio (2:1) were the exactly same for both session A and B. It could happen by a nonlinear feature of the IgA secretion depending on the duration of the task: IgA secretion rate might increase depending on the duration of the task. The change in the secretion rate would result in greater elevation of $\operatorname{IgA}$ concentration even taking an account of the difference in the duration of task period in session A, i.e. 18 minutes, and session B, 6 minutes. Subsequent recovering period was not enough in session A but appropriate in session B even it was equivalent in terms of task/break ratio. As such IgA secretion profile in the time series might reflect both the duration and repetition (or schedule) of the task/break. The idea just described was introduced into the kinetic mathematical model we proposed later (see section 5 for more detail).

With regard to the "sensitivity" against a mild stressor, IgA is not such sensitive to the onset or the end of a mild stressor as depicted in HF power profile (Fig. 6). However seen from another point, IgA secretion has cumulative feature, in other words it can represent a sort of hysteresis of a given stressor. Therefore $\operatorname{IgA}$ is not so sensitive against the temporal change in the situation, but it is still sensitive even for a mild stressor persisting certain time duration. In the field of dynamics, e.g. control engineering, it can be understood as the characteristic in the "time constant" or "relaxation time": in this case HF power possess small time constant against a mild stressor, and by contrast IgA possess relatively greater one. All together IgA can be a useful biomarker for a mild and long-lasting stressor.

\subsubsection{Profile of cortisol}

The cumulative secretion profile was more remarkable in the cortisol as depicted in Figure 8. As these figure shows salivary cortisol concentration showed a cumulative increase all through the task/break period in both session A and B. Moreover the elevated cortisol level did not recovered to the basal level despite of 20 minutes of recovering period after the sessions. HF power and saliva flow rate had no significant correlation with cortisol. It is understandable to think of that the secretion of cortisol reflects HPA system activity as already mentioned. 

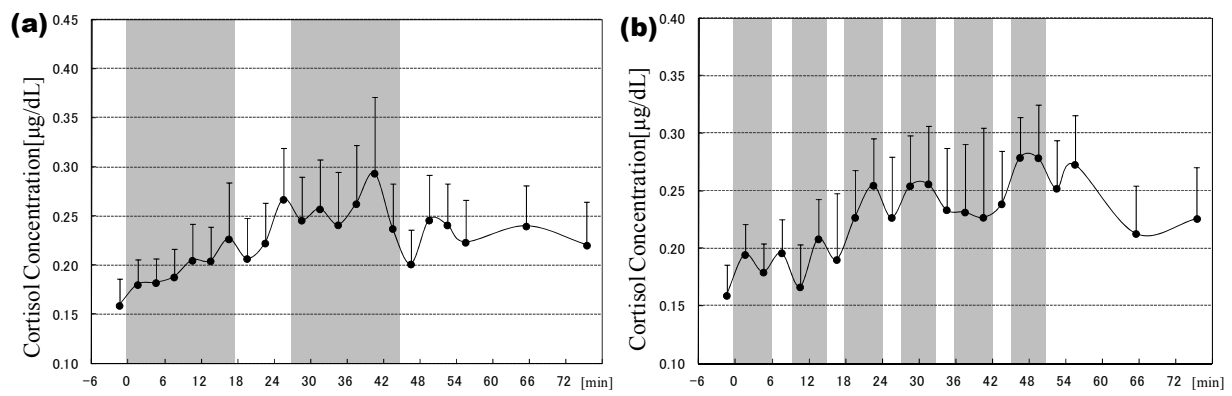

Fig. 8. Profile of cortisol in session A and B. Error bar represent standard error of the mean (S.E.M). Gray background represents a calculation task period.

With regard to the "sensitivity" against a mild stressor, it can be assumed by our experimental results that cortisol would possess greater time constant than that of $\operatorname{IgA}$, then greater than HF power of course. Comparing with the profile of IgA in the session B, cortisol still showed a cumulative effect in such a short-period of but repetition of the simple calculation task. In this sense, cortisol should be much sensitive than IgA against a mild stressor.

In the past cortisol studies, the stress response of cortisol against "mild" stressors, which are typified by the cognitive tasks without any threat or performance pressure such as passive stroop task and mental arithmetic task, showed inconsistent results, while that against acute and strong stressors such as academic oral defense with psychosocial evaluation always resulted in the increase of cortisol. Then a review article concluded that cortisol can be a useful stress biomarker for an acute and stronger stressor with psychosocial evaluation rather than a mild stressor (Dickerson, 2004). However looking into the result of our experiment in which the precise changing in the level of salivary cortisol were assessed every 3 minutes, a significant stress response can be observed even by a mild stressor if only salivary cortisol was assessed by an appropriate timing and duration corresponding to a particular targets.

\subsubsection{Profile of $\mathrm{CgA}$}

Figure 9 shows the profile of changing in the concentration of salivary secreted CgA during task/break period in the time series in the session A (Fig. 9(a)) and B (Fig. 9(b)). It increased in the task period and decreased in the break. Moreover both salivary flow rate and HF power, which represent parasympathetic nervous activities, have no significant correlation between $\mathrm{CgA}$. So $\mathrm{CgA}$ can also be taken as a plausible biomarker for a mild stressor.

The transient increase of $\mathrm{CgA}$ against laboratory stressors were also observed in the past $\mathrm{CgA}$ studies (Miyakawa, 2006; Kanamaru, 2006; Kanamaru, 2005). However some studies showed inconsistent results (e.g., Yamakoshi, 2009). It might attributes to the nature of high "temporal sensitivity" of CgA against a mild stressor illustrated in our study. As Fig. 9(a) shows CgA seems to possess a certain time constant, however it might be smaller than that of IgA even a little greater than that HF power. This means the slight difference in the saliva sampling time and duration might result in a big difference in the level of $\mathrm{CgA}$ in some cases. 

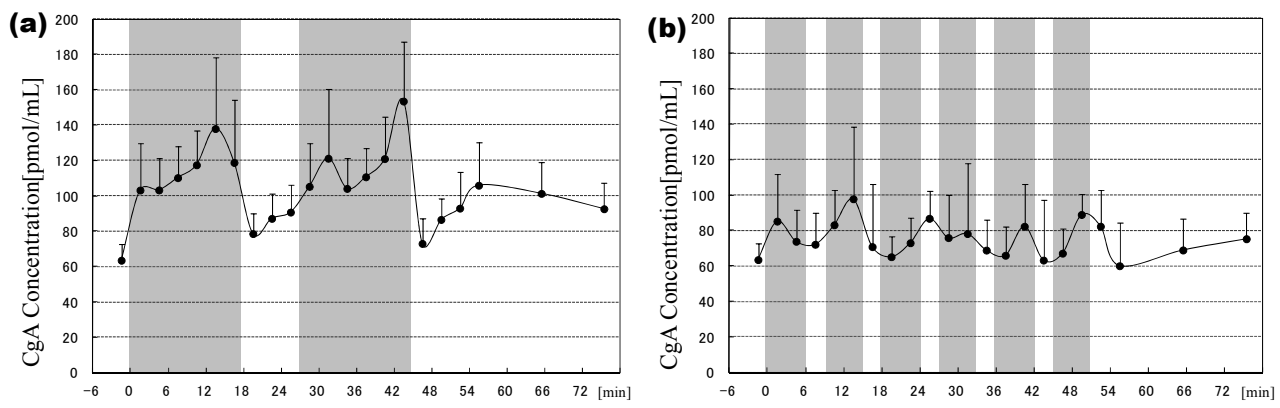

Fig. 9. Profile of CgA in session A and B. Error bar represent standard error of the mean (S.E.M). Gray background represents a calculation task period.

CgA possesses high "temporal sensitivity" as just described. On the other hand there could not find any cumulative effects like as HF power. Then it can be assumed that CgA possesses relatively small time constant. Since $\mathrm{CgA}$ is considered to represent sympathetic nervous system activity and thus to be a possible biomarker for SAM system activity as described in the subsection 2.3, this small time constant of CgA illustrated in our experiment is reasonable. So $\mathrm{CgA}$ can be a useful biomarker for detecting the change in the situation, i.e. the onset or the end of a given stressors but not suitable for estimating cumulative effect over time.

\subsubsection{Profile of DHEA}

The stress-induced secretion of salivary DHEA in session A and B seem to be cumulative as shown Figure 10(a) and 10(b). Moreover elevated DHEA during the both sessions did not return to the basal level during 20 minutes of recovering period, while total secretion of DHEA was larger in session A than B. Parasympathetic nervous activities indexed as HF power and saliva flow rate does not have significant correlation. There were some studies demonstrated that stress-induced DHEA secretion had reached its peak around 20 minutes after the onset of a strong stressor, TSST (Izawa, 2008; Sugaya, 2007). On the other hand looking at the result of our experiment assessing precise chaining of DHEA secretion in the time series, there might have a peak in DHEA in the last half of the second task period or much later in session A. Considering the difference in the nature of TSST and the calculation task, it can be assumed that the level of DHEA might represents the intensity of a given stressor, as the same as cortisol.

Comparing the profile of DHEA and other biomarkers, DHEA might possess relatively greater time constant: which should be ranked between that of $\operatorname{IgA}$ and cortisol. Therefore regarding the sensitivity of DHEA as a stress biomarker, it is not so sensitive to the onset or the end of the stressor, however it can still be a useful biomarker for long-lasting and a mild stressor like as cortisol. Since DHEA is a biomarker representing HPA system activity, it is understandable for that the stress response of DHEA was similar with cortisol. However there seems to be still a slight difference of response over time. It should be a matter in discussion in future. 

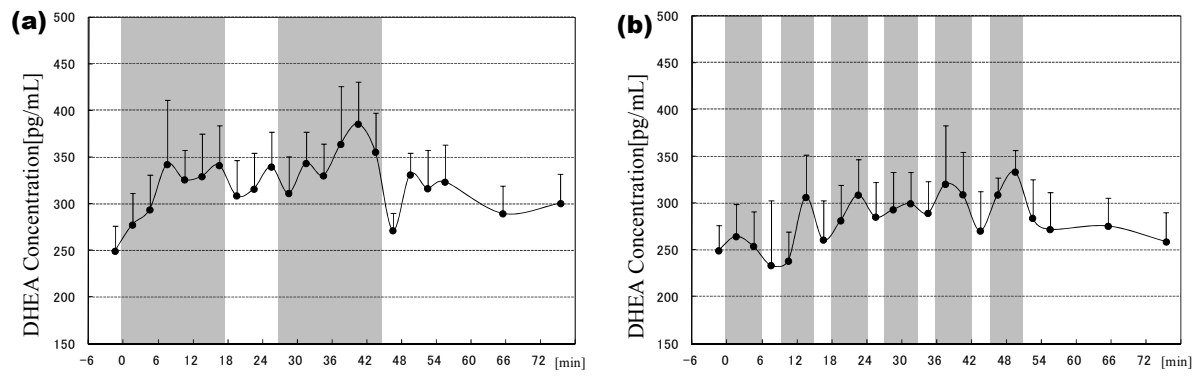

Fig. 10. Profile of DHEA in session A and B. Error bar represent standard error of the mean (S.E.M). Gray background represents a calculation task period.

\subsection{Discussion of the experiment: "Sensitivity" of the biomarkers as interpreted by the time constant; SAM comes first and HPA in the later}

To our knowledge our experiment was the first one to simultaneously illustrate the precise changing in the level of four stress biomarkers, which are IgA, cortisol, CgA, and DHEA, against a mild stressor. The results of our experiment plausibly demonstrate the possible candidacy of these substances as a biomarker for a mild laboratory stressor. Moreover the differences in the "sensitivity" among them as interpreted by the time constant were successfully demonstrated. The substances represents SAM system which are CgA and IgA sensitively increase and decrease by the onset and the end of the stressor, whereas those represents HPA system which are DHEA and cortisol showed cumulative effect over time. So our results might represent a part of complex dynamics of two major physiological stress reaction pathways, which are SAM and HPA. Moreover there also seems to be a difference in the time constant among SAM and HPA biomarker as CgA has the smallest, IgA places in the next, DHEA comes after IgA, and cortisol is the one which has the greatest time constant. It should represent further mechanism underlying the complexity of these systems and might be as a result of adaptive response in the sense of long evolutionary history: since cortisol possess a great impact on human physiology such as controlling blood pressure, it must be inefficient in term of energy consumption if it were as "sensitive" as CgA. In other word, it is of no use in responding a mild laboratory stressor but it should work in more critical situation in our life.

\section{Kinetic model of biomarkers in the response to a mild laboratory stressor: A preliminary description}

The difference in the sensitivity of stress biomarkers observed in our experiment can be restate as the difference in the time constant as described above. Here a mathematical model of the response of biomarkers is suggested to describe the experimental result as the difference in the time constant: from on/off binary response to cumulative one.

\subsection{Constitution of the kinetic model}

Basic assumptions for the model are introduced according to our experimental fact, and are quite simple as follows: 
1. Continuity: biomarker changes continuously in the time series,

2. Homeostasis: biomarker secretion has its upper and lower limits, and

3. Stress response: biomarker increases against stressor, and its rate is linearly depend on the duration of the stressor, which means non-linear increase of $\operatorname{Ig} \mathrm{A}$

4. Recovering: biomarker decrease to the basal level after the removal of the stressor.

As the most simple and well-consistent with these assumptions, the logistic function is adopted as the basis of this model. The logistic function is a nonlinear ordinary differential equation consisting of the first order of exponential increasing term and the second order of nonlinear decreasing term, as in

$$
d x / d t=(a-b x) x
$$

where $x, t, a$, and $b$ are all positive values representing the concentration of biomarker, time, and increasing and decreasing coefficient respectively. This formula is called a logistic function or growth curve, and it has been applied to describe the exponential growth in the number of bacteria and its decaying, caused by environmental deterioration accompanying
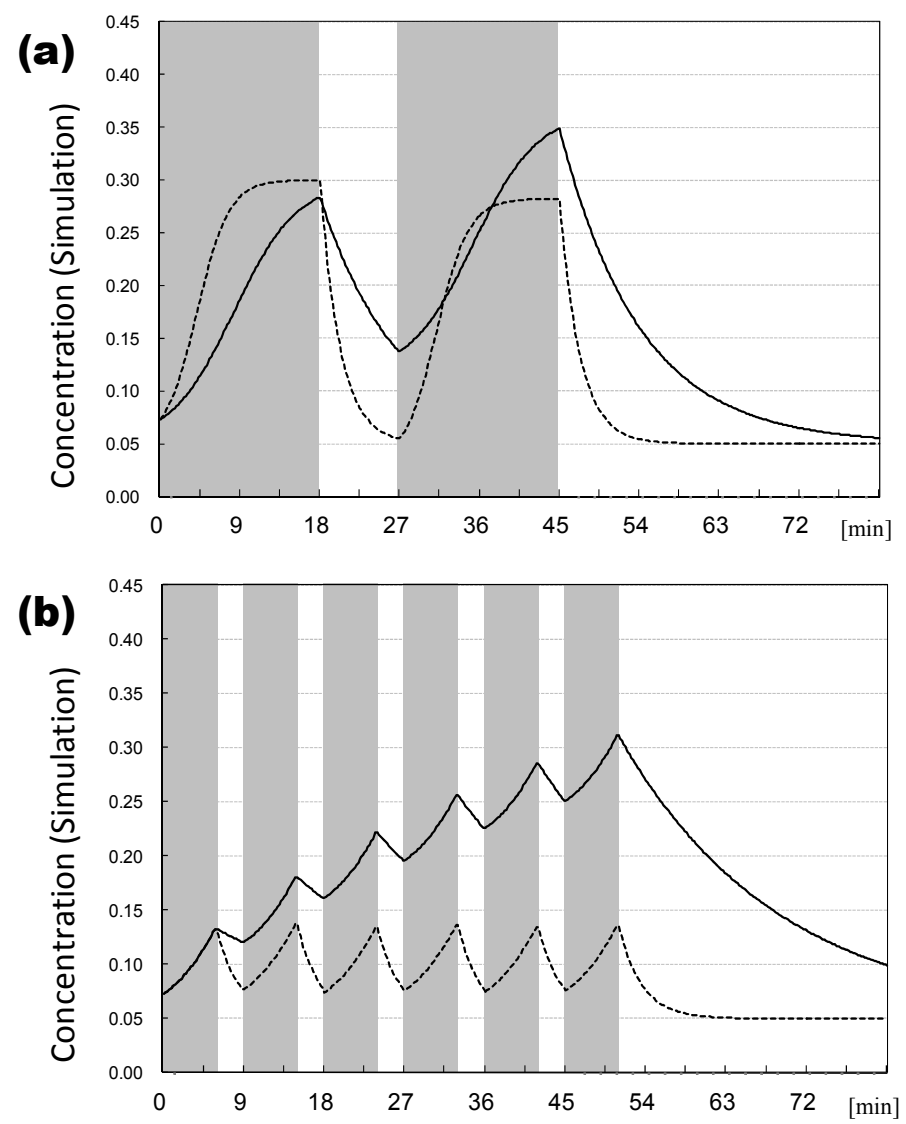

Fig. 11. Simulated biomarker secretion in session A and B. 
with an increase of individual density. Moreover, it is the simplest model possessing the homeostatic property. With regard to the recovering process, a simple exponential decreasing function is introduced, as in

$$
\mathrm{dx} / \mathrm{dt}=-\mathrm{cx}
$$

where $c$ is a positive values representing decreasing coefficient. The stress-induced response of biomarkers, increase by a short-term laboratory stressor and decrease by its removal, was simulated with task/break schedule as the same as in the session A and B in our experiment.

\subsection{Result and discussion of the numerical simulation}

Figure 11(a) and 11(b) shows the results of the simulation. As expected the model successfully illustrated on/off or cumulative changing profile of biomarkers depending on the parameters. By solely changing the increasing and decreasing parameters, $a, b$, and $c$, the degree of such cumulative effects was able to controlled. Therefore this simple non-linear kinetic model proposed here can be assumed as a basis for the stress induced physiological response in our body. By elaborating this model though a series of experiments targeting on the variety of stressors with different schedule on various biomarkers, the dynamics of human stress reaction pathways, HPA and SAM systems, would be better understood.

\section{Conclusion}

In this chapter, the salivary biomarker researches as a new metric for human mental stress, its background, methods, experiments, and kinetic model approaches were introduced. Although there are numbers of technical limitation and problems to be solved, biomarkers introduced in this manuscript can be useful and unique measures for human mental states. Stress estimation by salivary biomarkers has a great methodological advantage, because saliva can be collected less-stressfully and in a noninvasive manner unlike blood and urine. Moreover it is the one and the only secretory fluid that can be collected at anytime and by anyone including children and patients in need of nursing care.

On the other hand, mathematical model approach lead us an idea of the estimation of optimal work/break schedule in the limited time avoiding excessive secretion of biochemical substances: for an instance, when one has to take a long-distance drive and reach at a destination within a limited period of time, one could estimate the optimal timing of the stop for the rest. It might be useful for a stress management in the working place as well, such as VDT workload and monitoring work. The molecular analysis techniques are advancing day by day, real-time monitoring of such a tiny amount of biomarkers might be available in the near future. Remember the difficulty of self-management of stress and the necessity of introducing objective criteria. Biomarkers introduced in this manuscript can be a possible solution, even though it still remains in the initial step.

\section{Acknowledgment}

This study was supported in part by "Program to Disseminate Tenure Tracking System", Promoting Science and Technology of the Ministry of Education, Culture, Sports, Science and Technology, Japan. 


\section{References}

Ader, R.; Felten, L. \& Cohen, N. (Ed(s.). (2001) Psychoneruoimmunology (3rd ed.), Academic Press, Waltham, Massachusetts, USA

Ali, N. \& Pruessner, J.C.. (2011). The Salivary Alpha-amylase Over Cortisol Ratio as a Marker to Assess Dysregulations of the Stress Systems. Physiology \& Behavior, [Epub ahead of print]

Andreassi, J.L. (2007). Psychophysiology: Human Behavior \& Physiological Response (5th ed.), Psychology Press, London, England

Bosch, J.A.; Ring, C.; de Geus, E.J.; Veerman, E.C. \& Amerongen, A.V. (2002). Stress and secretory immunity. International Review of Neurobiology, Vol.52, No.1, pp.213-253

Buchsbaum, M.S.; Muscettola, G. \& Goodwin, F.K. (1981). Urinary MHPG, stress response, personality factors and patients with major affective disorders. Neuropsychology,Vol.7, pp.212-224

Clow, A.; Thorn, L.; Evans, P. \& Hucklebridge, F. (2004). The awakening cortisol response: methodological issues and significance. Stress, Vol.7, No.1, pp.29-37

Deguchi, M.; Wakasugi, J.; Ikegami, T.; Nanba, S. \& Yamgaguchi, M. (2006). Evaluation of driver stress using motor-vehicle driving simulator. IEEJ Trans. Sensors and Micromachines, Vol.126, No.8, pp.438-444

Dickerson, S.S. \& Kemeny, M.E. (2004). Acute stressors and cortisol responses: A theoretical integration and synthesis of laboratory research. Psychological Bulletin, Vol.130, No.3, pp.335-391

Evans, P.; Bristow, M.; Hucklebridge, F.; Clow, A. \& Pang, F.Y. (1994). Stress, arousal, cortisol and secretory Immunoglobulin A in students undergoing assessment. The British journal of clinical psychology, Vol.33, pp.575-576

Gabriel, P. \& Liimatainen, M.R. (2000). Mental health in the workplace. International Labor Organization. 10.11.2011, Available from http://ilo.org

Green, R.G. \& Green, M.L. (1987). Relaxation increases salivary Immunoglobulin A. Psychological Reports, Vol.61, pp.623-629

Gregory, R.L.; Kim, D.E.; Kindle, J.C.; Hobbs, L.C. \& Lloyd, D.R. (1992). Immunoglobulindegrading enzymes in localized juvenile periodontitis. Journal of periodontal research, Vol.27 No.3, pp.176-183

Hansen, A.M.; Garde, A.H. \& Persson, R. (2008). Source of biological and methodological variation in salivary cortisol and their impact on measurement among healthy adults: A review. The Scandinavian Journal of Clinical $\mathcal{E}$ Laboratory Investigation, Vol.68, No.6, pp.448-458

Hellhammer, D.H.; Wüst, S. \& Kudielka, B.M. (2009). Salivary cortisol as a biomarker in stress research. Psychoneuroendocrinology, Vol.34, No.2, pp.163-171

Hucklebridge, F.; Hussain, T.; Evans, P. \& Clow, A. (2005). The diurnal patterns of the adrenal steroids cortisol and dehydroepiandrosterone (DHEA) in relation to awakening; Psychoneuroendocrinology, Vol.30, No.1, pp.51-57

Isowa, T.; Ohira, H. \&d Murashima, S. (2004). Reactivity of immune, endocrine and cardiovascular parameters to active and passive acute stress. Biological Psychology, Vol.65, pp.101-120

Izawa, S.; Sugaya, N.; Shirotsuki, K.; Yamada, K.C.; Ogawa, N.; Ouchi, Y.; Nagano, Y.; Suzuki, K. \& Nomura, S. (2008). Salivary Dehydroepiandrosterone secretion in 
response to acute psychosocial stress and its correlations with biological and psychological changes. Biological Psychology, Vol.79, No.3, pp.294-298

Izawa, S.; Sugaya, N.; Ogawa, N.; Nagano, Y.; Nakano, M.; Nakase, E.; Shirotsuki, K.; Yamada, K. C.; Machida, K.; Kodama, M. \& Nomura, S. (2007). Episodic stress associated with writing a graduation thesis and free cortisol secretion after awakening. International Journal of Psychophysiology, Vol.64, No.2, pp.141-145

Izawa, S.; Shirotsuki, K.; Sugaya, N.; Ogawa, N.; Suzuki, K. \& Nomura, S. (2004). The application of saliva to an assessment of stress: procedures for collecting and analyzing saliva and characteristics of salivary substances. Japanese Journal of Complementary and Alternative Medicine, Vol.4, No.3, pp.91-101

Jemmott III, J.B.; Borysenko, J.Z.; Borysenko, M.; McClelland, D.C.; Chapman, R.; Meyer, D. \& Benson, H. (1983). Academic stress, power motivation, and decrease in secretion rate of salivary secretory Immunoglobulin A. Lancet, Vol.321, No.8339, pp.14001402

Jemmott III, J.B. \& Magloire, K. (1988). Academic stress, social support, and secretory Immunoglobulin A. Journal of Personality and Social Psychology, Vol.55, No.5, pp.803810

Jemmott III, J.B. \& McClelland, D.C. (1989). Secretory IgA as a measure of resistance to infectious disease: comments on Stone, Cox, Valdimarsdottir, and Neale. Behavioral Medicine, Vol.15, pp.63- 71

Kanamaru, Y.; Kikukawa, A. \& Shimamura, K. (2006). Salivary chromogranin-A as a marker of psychological stress during a cognitive test battery in humans. Stress,. Vol.9, No.3, pp.127-131

Kim, Y. \& Loh, Y.P. (2005). Chromogranin A: a surprising link between granule biogenesis and hypertension. J Clin Invest. Vol.115, No.7, pp.1711-1713

Kimura, K.; Isowa, T.; Ohira, H. \& Murashima, S. (2005). Temporal variation of acute stress responses in sympathetic nervous and immune systesm. Biological Psychology, Vol.70, pp.131-139

Kirschbaum, C.; Pirke, K.M. \& Hellhammer, D.H. (1993). The 'Trier Social Stress Test' -a tool for investigating psychobiological stress responses in a laboratory setting. Neuropsychobiology. Vol.28, No.1-2, pp.76-81

Kirschbaum, C. \& Hellhammer, D.H. (1994). Salivary cortisol in psychoneuroendocrine research: recent developments and applications. Psychoneuroendocrinology, Vol.19, No.1, pp.313-333

Knight, W.E. \& Rickard, N.S. (2001). Relaxing music prevents stress-induced increase in subjective anxiety, systolic blood Pressure, and heart rate in healthy males and females. Journal of Music Therapy,Vol.38, pp.254-272

Martin, R.A. \& Dobbin, J.P. (1988). Sense of humor, hassles, and Immunoglobulin A: evidence for a stress-moderating effect of humor. International journal of psychiatry in medicine, Vol.18, pp.93-105

Martin, R.B.; Guthrie, C.A. \& Pitts, C.G. (1993). Emotional crying, depressed mood, and secretory Immunoglobulin A. Behavioral Medicine, Vol.19, No.3, pp.111-114

McNair, M.; Heuchert, P. \& Shilony, E. (2003). Profile of mood states Bibliography 1964-2002. Multi-Health-Systems Inc, North Tonawanda, NewYork 
Michael, A.; Jenaway, A.; Paykel, E.S. \& Herbert, J. (2000). Altered salivary Dehydroepiandrosterone levels in major depression in adults. Biological Psychiatry, Vol.48, No.10, pp.989-995

Miyakawa, M.; Matsui, T.; Kishikawa, H.; Murayama, R.; Uchiyama, I.; Itoh, T. \& Yoshida, T. (2006). Salivary Chromogranin A as a measure of stress response to noise. Noise Health. Vol.8, No.32, pp.108-113

Morgan, C.A. 3rd.; Rasmusson, A.; Pietrzak, R.H.; Coric, V. \& Southwick, S.M. (2009). Relationships among plasma dehydroepiandrosterone and dehydroepiandrosterone sulfate, cortisol, symptoms of dissociation, and objective performance in humans exposed to underwater navigation stress; Biological Psychiatry., Vol.66, No.4, pp.334-340

Nakane, H. (1999). Salivary Chromogranin A as index of psychosomatic stress response. RED Review of Toyota CRDL., Vol.34, No.3, pp.17-22

Nomura, S.; Tanaka, H. \& Moriyama, T. (2006). Pilot Study of SIgA as a Stress Maker with Repetitive Saliva Collection, Proceedings of the 5th International Conference of the Cognitive Science, pp.169-170, Vancouver, Canada, July 26-29, 2006

Nomura, S.; Mizuno, T.; Nozawa, A.; Asano, H. \& Ide, H. (2009). Salivary cortisol as a possible physiological biomarker for mild mental workload. Biofeedback Research, Vo.36, No.1, pp.23-32

Nomura, S.; Mizuno, T.; Nozawa, A.; Asano, H. \& Ide, H. (2010a). Characteristics of Salivary Chromogranin A as a Short-term Mental Stress Biomarker. Journal of Biomedical Engineering, Vol.48, No.2, pp.207-212

Nomura, S.; Mizuno, T.; Nozawa, A.; Asano, H. \& Ide, H. (2010b). Salivary DHEA as a Possible Stress-biomarker for a Mild Mental Workload. Transactions of Human Interface Society, Vol.12, No.3, pp.33-40

Noto, Y.; Sato, T.; Kudo, M.; Kurata, K. \& Hirota, K. (2005). The relationship between salivary biomarkers and State-Trait Anxiety Inventory score under mental arithmetic stress: a pilot study. Anesth Analg., Vol.101, pp.1873-1876

Ockenfels, M.C.; Porter, L.; Smyth, J.; Kirschbaum, C.; Hellhammer, D.H. \& Stone, A.A. (1995). Effect of chronic stress associated with unemployment on salivary cortisol: overall cortisol levels, diurnal rhythm, and acute stress reactivity. Psychosomatic Medicine, Vol.57, No.5, pp.460-467

Ohira, H. (2004). Social support and salivary secretory Immunoglobulin A response in women to stress of making a public speech. Perceptual and Motor Skills, Vol.98, pp.1241-1250

Ohira, H. (2001). Controllability of aversive stimuli unconsciously determines volume of secretory Immunoglobulin A in saliva. Japanese Journal of Behavioral Medicine, Vol.6, pp.16-28

Ohira, H.; Watanabe, Y.; Kobayashi, K. \& M. Kawai. (1999). The Type A behavior pattern and immune reactivity to brief stress: change of volume of secretory Immunoglobulin A in saliva. Perceptual and Motor Skills, Vol.89, pp.423-430

Okamura, H.; Tsuda, A.; Yajima, J.; Mark, H.; Horiuchi, S.; Toyoshima, N. \& Matsuishi, T. (2010). Short Sleeping Time and Psychobiological Responses to Acute Stress. International Journal of Psychophysiology, Vol.78, No.3, pp.209-214

Powell, L.H.; Lovallo, W.R.; Matthews, K.A.; Meyer, P.; Midgley, A.R.; Baum, A.; Stone, A.A.; Underwood, L.; McCann, J.J.; Janikula Herro, K. \& Ory, M.G. (2002). 
Physiologic markers of chronic stress in premenopausal, middle-aged women. Psychosomatic Medicine, Vol.64, No.3, pp.502-509

Pruessner, J.C.; Wolf, O.T.; Hellhammer, D.H.; Buske-Kirschbaum, A.; von Auer. K.; Jobst. S.; Kaspers. F. \& Kirschbaum, C. (1997). Free cortisol levels after awakening: a reliable biological marker for the assessment of adrenocortical activity. Life Sci. Vol.61, pp.2539-2549

Schoofs, D. \& Wolfa, O.T. (2011) Are Salivary Gonadal Steroid Concentrations Influenced by Acute Psychosocial Stress? A Study Using the Trier Social Stress Test (TSST). International Journal of Psychophysiology, Vol.80, No.1, pp.36-43

Schulz, P.; Kirschbaum, C.; Prüßner, J. \& Hellhammer, D. (1998). Increased free cortisol secretion after awakening in chronically stressed individuals due to work overload. Stress Med. Vol.14, No.2, pp.91-97

Shirtcliff, E.; Zahn-Waxler, C.; Klimes-Dougan, B. \& Slattery, M. (2007). Salivary dehydroepiandrosterone responsiveness to social challenge in adolescents with internalizing problems. J Child Psychol Psychiatry., Vol.48, No.6, pp. 580-591

Steptoe. A.; Cropley. M.; Griffith. J. \& Kirschbaum, C. (2000). Job strain and anger expression predict early morning elevations in salivary cortisol. Psychosomatic Medicine, Vol.62, No.2, pp.286-292

Steptoe, A. \& Wardle, J. (2005). Positive affect and biological function in everyday life. Nuerobiology of Aging, Vol.26, No.1, pp.108-112

Strahler, J.; Berndt, C.; Kirschbaum, C. \& Rohleder, N. (2010). Aging Diurnal Rhythms and Chronic Stress: Distinct Alteration of Diurnal Rhythmicity of Salivary a-amylase and Cortisol. Biological Psychology, Vol.84, No.2, pp.248-256

Stone, A.A.; Neale, J.M.; Cox, D.S.; Napoli, A.; Valdimarsdottir, H. \& Kennedy-Moore, E. (1994). Daily events are associated with a secretory immune response to an oral antigen in men. Health Psychology, Vol.13, No.5, pp.440-446

Sugaya, N.; Izawa, S.; Ouchi, Y.; Shirotsuki,K.; Yamada, K.C.; Ogawa, N.; Nagano, Y. \& Nomura, S. (2007). Adrenal and Autonomic Responses to Psychosocial Stress in Individuals with Irritable Bowel Syndrome. Japanese Journal of Psychosomatic Medicine, Vol.47, No.12, pp.1013-1022

Toda, M.; Kusakabe, S.; Nagasawa, S.; Kitamura, K. \& Morimoto, K. (2007). Effect of laughter on salivary endocrinological stress marker Chromogranin A. Biomed Res., Vol.28, No.2, pp.15-18

Tsujita, S. \& Morimoto, K. (1999). Secretory IgA in saliva can be a useful stress maker. Environmental Health and Preventive Medicine, Vol.4, pp.1-8

Valdimarsdottir, H.B. \& Stone, A.A. (1997). Psychosocial factors and secretory Immunoglobulin A. Critical Reviews in Oral Biology \& Medicine, Vol.8, No.4, pp.461474

van Stegeren, A.; Rohleder, N.; Everaerd, W. \& Wolf, O.T. (2006). Salivary alpha amylase as marker for adrenergic activity during stress: effect of betablockade. Psychoneuroendocrinology, Vol.31, No.1, pp.137-141

Wakida, S.; Tanaka, Y. \& Nagai., H. (2004). High throughput screening for stress marker. Bunseki, Vol.2004, pp.309-316

Williams, R.B. (1986). Patterns of reactivity and stress; Handbook of stress, reactivity, and cardiovascular disease, John Wiley \& Sons, Hoboken, New Jersey, USA 
Wingernfeld, K.; Schulz, M.; Damkroeger, A.; Philippsen, C.; Rose, M. \& Driessen, M. (2010). The Dirunal Course of Salivary Alpha-amylase in Nurses: An Investigation of Potential Confounders and Associations with Stress. Biological Psychology, Vol.85, No.1, pp.179-181

Winkler, H. \& Fischer-Colbrie, R. (1992). The chromogranins A and B: the first 25 years and future perspectives. Neuroscience, Vol.49, No.3, pp.497-528

Wolf, O.T. \& Kirschbaum, C. (1999). Actions of Dehydroepiandrosterone and its sulfate in the central nervous system: Effects on cognition and emotion in animals and humans. Brain Res Rev., Vol.30, No.3, pp.264-288

Yamaguchi, M., Kanemori, T., Knemaru, M., Takai, N., Yasufumi, M. \& Yoshida, H. (2004). Performance evaluation of salivary amylase activity monitor. Biosens.Bioelectron., Vol.20, pp.491-497

Yamakoshi, T.; Park, S.B.; Jang, W.C.; Kim, K.; Yamakoshi, Y. \& Hirose, H. (2009). Relationship between salivary chromogranin-A and stress induced by simulated monotonous driving. Med Biol Eng Comput., Vol.47, pp.449-456.

Yokoyama, K. \& Araki, S. (1993). Nihongo ban POMS tebiki (the guide of profile of mood states Japanese version, 5th ed.), Kaneko Shobo, Tokyo, Japan 


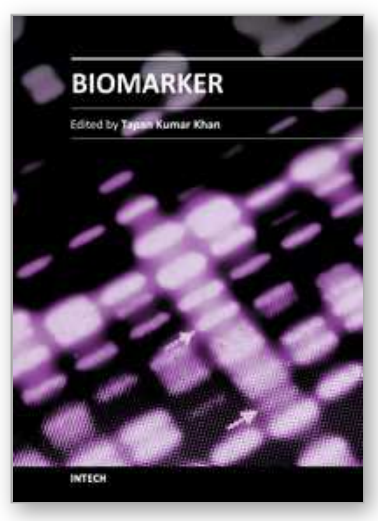

\author{
Biomarker \\ Edited by Prof. Tapan Khan
}

ISBN 978-953-51-0577-0

Hard cover, 392 pages

Publisher InTech

Published online 27, April, 2012

Published in print edition April, 2012

Clinicians, scientists, and health care professionals use biomarkers or biological markers as a measure of a person's present health condition or response to interventions. An ideal -biomarker should have the following criteria: (I) ability to detect fundamental features of the disease, (II) ability to differentiate from other closely related diseases, (III) ability to detect early stages and stages of progression, (IV) the method should be highly reliable, easy to perform and inexpensive, and (V) sample sources should be easily accessible from body. Most of the chapters in this book follow the basic principle of biomarkers.

\title{
How to reference
}

In order to correctly reference this scholarly work, feel free to copy and paste the following:

Shusaku Nomura (2012). Salivary Hormones, Immunes and Other Secretory Substances as Possible Stress Biomarker, Biomarker, Prof. Tapan Khan (Ed.), ISBN: 978-953-51-0577-0, InTech, Available from: http://www.intechopen.com/books/biomarker/salivary-hormones-immunes-and-other-secretory-substances-asa-possible-stress-biomarker

\section{INTECH}

open science / open minds

\section{InTech Europe}

University Campus STeP Ri

Slavka Krautzeka 83/A

51000 Rijeka, Croatia

Phone: +385 (51) 770447

Fax: +385 (51) 686166

www.intechopen.com

\section{InTech China}

Unit 405, Office Block, Hotel Equatorial Shanghai

No.65, Yan An Road (West), Shanghai, 200040, China 中国上海市延安西路65号上海国际贵都大饭店办公楼 405 单元

Phone: +86-21-62489820

Fax: +86-21-62489821 
(C) 2012 The Author(s). Licensee IntechOpen. This is an open access article distributed under the terms of the Creative Commons Attribution 3.0 License, which permits unrestricted use, distribution, and reproduction in any medium, provided the original work is properly cited. 